\title{
MR connectomics: Principles and challenges
}

\author{
Patric Hagmann ${ }^{\text {a,b,c,* }}$, Leila Cammoun ${ }^{b}$, Xavier Gigandet ${ }^{b}$, Stephan Gerhard ${ }^{b}$, P. Ellen Grant ${ }^{c, d}$, \\ Van Wedeen $^{d}$, Reto Meuli ${ }^{a}$, Jean-Philippe Thiran ${ }^{b}$, Christopher J. Honey ${ }^{e}$, Olaf Sporns ${ }^{f}$ \\ a Department of Radiology, University Hospital Center and University of Lausanne (CHUV-UNIL), Switzerland \\ b Signal Processing Laboratory (LTS5), Ecole Polytechnique Fédérale de Lausanne (EPFL), Switzerland \\ c Division of Newborn Medicine, Department of Medicine and Department of Radiology, Children's Hospital Boston, USA \\ d Athinoula A. Martinos Center for Biomedical Imaging, Member of the Affiliated Faculty of the Harvard-MIT Division of Health Sciences and Technology, Charlestown, MA, USA \\ e Department of Psychology, Green Hall, Princeton University, Princeton, NJ, USA \\ ${ }^{\mathrm{f}}$ Department of Psychological and Brain Sciences, Indiana University, Bloomington, IN, USA
}

\section{A R T I C L E I N F O}

\section{Article history:}

Received 31 August 2009

Received in revised form 2 January 2010

Accepted 13 January 2010

\section{Keywords:}

Diffusion MRI

Tractography

Networks

Connectivity

Validation

Resting-state fMRI

Database

\begin{abstract}
A B S T R A C T
MR connectomics is an emerging framework in neuro-science that combines diffusion MRI and whole brain tractography methodologies with the analytical tools of network science. In the present work we review the current methods enabling structural connectivity mapping with MRI and show how such data can be used to infer new information of both brain structure and function. We also list the technical challenges that should be addressed in the future to achieve high-resolution maps of structural connectivity. From the resulting tremendous amount of data that is going to be accumulated soon, we discuss what new challenges must be tackled in terms of methods for advanced network analysis and visualization, as well data organization and distribution. This new framework is well suited to investigate key questions on brain complexity and we try to foresee what fields will most benefit from these approaches.
\end{abstract}

(c) 2010 Elsevier B.V. All rights reserved.

\section{Introduction}

We entered the twenty first century with the capacity to map any person's individual genetic profile (Gresham et al., 2008; Hutchison, 2007). This genomic information serves, at individual and at population levels, as a structural scaffold that helps us understanding, characterizing and predicting normal and pathologic function at multiple levels like the transcriptional, proteomic, cellular and systemic level (Mo and Palsson, 2009). The emergence of systems biology at all these levels has directly emerged from our ability to map not only the individual genes but also the complex interactions between them as well as the highly variable posttranscription modifications.

The development of chemical tracing technologies over the past 60 years has provided us with a relatively good view of the mesoscopic architecture of non-human white matter fiber pathways (Schmahmann and Pandya, 2006). However, progress has been hampered by many technological difficulties and low throughput. Furthermore variability is difficult to assess since only a limited set of connections can be traced per specimen. Therefore, even for the

\footnotetext{
* Corresponding author at: Department de Radiologie Médicale, Rue du Bugnon 46, 1011 Lausanne-CHUV, Switzerland. Tel.: +41213141111.

E-mail address: Patric.hagmann@epfl.ch (P. Hagmann).
}

brain of the heavily studied macaque, our connectional map has not yet attained a macroscopic completeness. Surveying this state of affairs about 15 years ago, Crick and Jones called for the development of radically new techniques to investigate brain connectivity (Crick and Jones, 1993).

Today, Crick's and Jones' call seems to be receiving a rather strong echo that in several respects is summarized in this special edition. In addition to the important advances achieved in chemical tracing methods major initiatives are appearing to use information technology and computer science in order to collect data at highthroughput and to organize data in large databases (Bohland et al., 2009; Stephan et al., 2001). This is not only the case for chemical tracing technology but also for human in vivo imaging with the advent of diffusion MRI technology that has made tremendous progress over the last 10 years. As we will see, diffusion imaging has opened the door for high-throughput individual non-invasive in vivo whole brain structural connectivity mapping of the human brain by the inference of axonal fiber pathways from local water diffusion.

The perspective of collecting large amounts of connectional data combined with the understanding that the fundamental properties of the brain result from large-scale network topology has led two researchers, at that time independent, to realize the prime importance of this emerging technology, and to conceptualize in 2005 the notion of "connectome" and its related science 
“connectomics” (Hagmann, 2005; Sporns et al., 2005). In complete analogy to the word "genome", this new "-ome" emphasizes to the notion that the brain is one large and unique structural entity: a network made of neural connections (edges) and neural units (nodes). Depending on the scale, connections are axonal fiber pathways or individual axons and dendrites, and neural units are cortical functional units, cortical columns, or individual neurons. It also implies that the huge diversity of complex behaviors and brain properties is shaped by its global connectional organization. The word "connectomics" emphasizes that there is a new field of science and many technological challenges to be tackled around the "connectome", from mapping the nodes and edges in the brain at an individual and population level, developing the tools to analyze its organization and structure to understanding the interdependence with several levels of brain function.

In the present review we will first sketch the methods for mapping the connectome step by step using diffusion MRI. At each of these steps we will describe the state of the art and the remaining technical challenges. The following section is entirely dedicated to a discussion on the validation of diffusion MRI derived connectivity mapping, a particularly important and difficult problem. Then we will discuss the possible methods for analyzing brain structure and function using the connectome framework. Finally we will try to glance towards the future and imagine the tools that will be developed to serve the connectome project and what neuro-scientific questions may potentially benefit most form connectomics.

\section{Mapping the connectome with MRI}

\subsection{Diffusion imaging}

\subsubsection{Past and current state of the art}

Diffusion-weighted magnetic resonance imaging (MRI), received strong attention for the first time in the early 90 s as it proved to be a sensitive biomarker for acute stroke (Moseley et al., 1990). The second reason diffusion imaging came to attention, was because it was observed that not only does white matter exhibit reliable anisotropic properties (Basser et al., 1994; Douek et al., 1991) but that it is indeed possible to infer fiber tract trajectories on the basis of these patterns (Conturo et al., 1999; Mori et al., 1999; Wedeen, 1996). Diffusion Tensor Imaging and tractography was born. However relatively quickly people realized that major tract reconstruction artifacts were difficult to overcome (Tuch et al., 2002; Wiegell et al., 2000). These were related to the fact that DTI was unable to resolve multiple fiber bundle orientation inside an imaging voxel, and these crossing configurations appeared to be ubiquitous in the brain (Behrens et al., 2007). While the theoretical foundations of diffusion MRI have come to be better understood (Wedeen et al., 2005), over the years, increasingly complex data acquisition schemes have been developed that are able to handle "the fiber-crossing problem" (Tournier et al., 2004; Tuch et al., 2003; Wedeen et al., 2000, 2005).

Water molecules inside tissues, at body or room temperature, experience random motion due to thermal energy; this is commonly referred as Brownian motion (Einstein, 1956; Le Bihan, 1995). Over a limited volume, i.e. a voxel of a few $\mathrm{mm}^{3}$, the displacement of such a population of water molecules can be described conveniently as a displacement probability density function (PDF) (Callaghan, 1991). Obviously, the shape of the PDF is strongly influenced by the local environment (Fig. 1); i.e. the orientation of the local barriers created by cellular membranes, myelin sheaths, macromolecules, and so forth are directly reflected in the PDF. The shape of the PDF is also different in every position of the brain since local tissue architecture is location dependent (Beaulieu, 2002).

Diffusion imaging exploits the fact that motion of water molecules inside tissues can be magnetically labeled by the appli- cation of a strong bipolar gradient during image acquisition, for review see Le Bihan (2003). In fact, the diffusion MRI experiment produces a signal that is the Fourier transform of the diffusion PDF at every imaged brain location. Through this fundamental relationship between MR signal and PDF, it is possible to recover for every imaging voxel the local diffusion PDF, provided that the MR signal is appropriately sampled (Callaghan, 1991; Cory and Garroway, 1990; Wedeen et al., 2005).

This apparent simplicity in concept has conflicted and still conflicts with the technical requirements of such imaging. Indeed the direct implementation of the above-described theory is materialized by Diffusion Spectrum Imaging (DSI) (Wedeen et al., 2000, 2005), a technique that is characterized by the acquisition of a very large number of images applying strong motion sensitizing magnetic gradients in multiple directions. This not only results in lengthy acquisition times but also requires high performance hardware, typically 3 Tesla systems, powerful magnetic gradients and multichannel head coils. For these reasons, DSI is only now starting to play a significant role in brain imaging as more centers are equipped with high-end magnets and as commercially available pulse sequences are being distributed (Krueger, 2008). This is the reason why DTI, a much simpler scheme, is still largely in use. Therefore most connectional studies up to now have been conducted with DTI schemes despite its recognized limitations. Somewhat in-between solutions, known as single-shell High Angular Resolution Diffusion Imaging (HARDI) techniques became quite popular since through some assumptions they were able to overcome the "fiber-crossing problem" without having to compromise to much scan time and without major hardware requirements (Jansons and Alexander, 2003; Seunarine and Alexander, 2009; Tournier et al., 2004; Tuch et al., 2002, 2003). In our experience, there is a steady increase in performance from DTI to HARDI techniques and finally DSI when performed with the appropriate hardware, pulse sequence and post-processing (Gigandet, 2009; Wedeen et al., 2008), but this is not a broadly accepted fact. However all the techniques provide satisfying result to a given point only, and reconstruction errors are identifiable with every method.

\subsubsection{Challenges and perspectives}

From post mortem studies in macaque, we know that we are likely, with improved technology, to resolve structures that cannot be resolved yet with current in vivo scanning methods. Typically we know from such experiments that stronger diffusion sensitizing gradients than the ones available on current human scanners need to be developed and will require significant engineering effort. The reason for striving for stronger gradients is twofold, (a) it permits sampling of images at high $b$-values with shorter diffusion encoding times, which will improve angular resolution and (b) a shorter diffusion sensitizing gradient duration helps to decrease the echo time, which further boosts the SNR. Multichannel coil technology with at least 32 (and up to 128) channels will not only help to increase SNR but also reduce the acquisition time when combined with fast imaging methods such as parallel imaging and multiple slice encoding pulse sequences (Reese et al., 2009; Wiggins et al., 2009). This will ultimately allow increased spatial resolution and decrease the susceptibility artifacts inherent to echo planar imaging. The combination of these developments will be critical if we are to bring connectivity mapping to the next level, ultimately moving from the macro- to the meso-scale connectome resolution.

\subsection{MR tractography}

\subsubsection{Past and current state of the art}

Tractography is an algorithmic method for inferring the trajectories of fiber bundles through the white matter on the basis of diffusion MRI data. We have seen in the previous section how a diffusion 


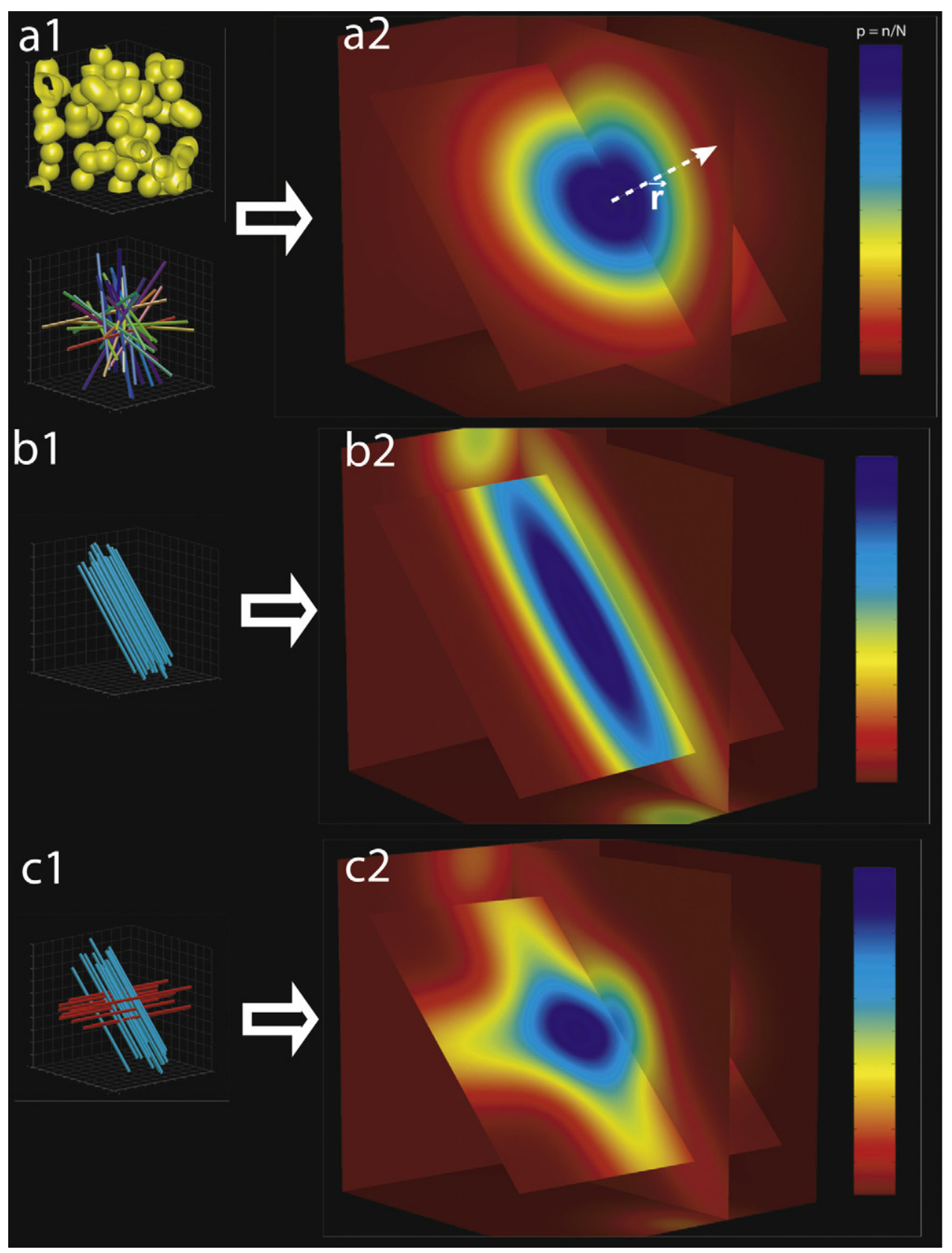

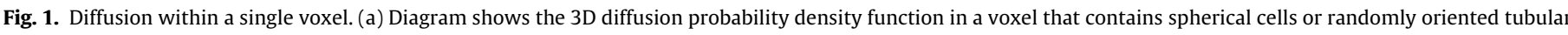

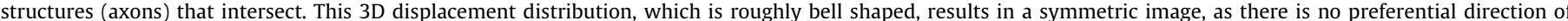

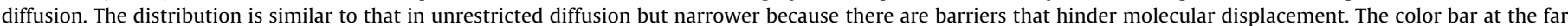

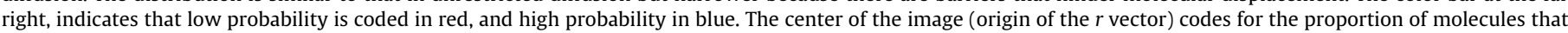

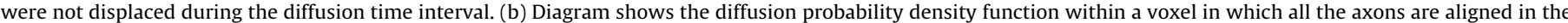

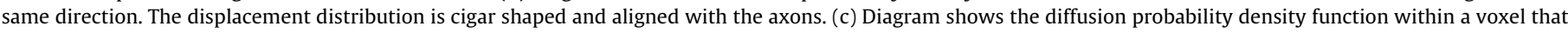

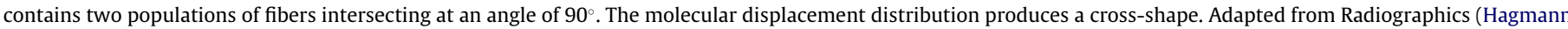
et al., 2006).

PDF can be measured in every voxel of white matter. From such PDF, directions of preferred or maximal diffusion can be extracted, i.e. directions in which the molecular displacement of water is faster that in others. Each voxel may exhibit one or several such principle diffusion directions. Each principal direction is mostly tangent locally to a fiber bundle. Therefore principal directions of diffusion are coherently aligned along white matter fiber tracts defining coherence paths of maximal diffusion. A tractography algorithm is a tool to capture this coherence and to map it as a curve in three-dimensional space, hence representing a fiber tract trajectory (Hagmann, 2005; Hagmann et al., 2007; Wedeen et al., 2008).
Tractography has captured the imagination of many computer scientists, and all sorts of possible solutions to tackle the problem have been investigated. To provide an overview for the lay reader, we will review the principle of the simplest and most straightforward algorithm, local path integration approach commonly known as "streamline" tractography.

In the setting of DTI, the dataset of principal diffusion directions can be considered as a three-dimensional vector field, similar to a weather wind map. The theoretical trajectory of a particle in such a wind map, i.e. diffusion map, can be computed by path integration, basically meaning that from a chosen point the particle follows a 
Table 1

Classification of major tractography algorithms published depending on diffusion imaging and propagation technique.

\begin{tabular}{|c|c|c|}
\hline Tractography method & DTI & HARDI \\
\hline \multicolumn{3}{|l|}{ Deterministic line propagation } \\
\hline $\begin{array}{l}\text { Local path integration along principal } \\
\text { diffusion directions }\end{array}$ & $\begin{array}{l}\text { Wedeen (1996), Conturo et al. (1999), Mori et al. (1999), } \\
\text { Basser et al. (2000). }\end{array}$ & Hagmann (2005), Hagmann et al. (2007), Wedeen et al. (2008). \\
\hline Tensor deflection & Lazar et al. (2003) & \\
\hline \multicolumn{3}{|l|}{ Probabilistic line propagation } \\
\hline Random walk simulation & $\begin{array}{l}\text { Koch et al. (2002), Hagmann et al. (2003), Parker et al. } \\
\text { (2003). }\end{array}$ & Parker and Alexander (2005), Perrin et al. (2005). \\
\hline Bayesian framework & Behrens et al. (2003) & Behrens et al. (2007). \\
\hline \multicolumn{3}{|l|}{ Energy minimization techniques } \\
\hline Dijkstra’s algorithm & Iturria-Medina et al. (2007) & \\
\hline Fast marching & $\begin{array}{l}\text { Parker et al. (2002), Staempfli et al. (2006), Jbabdi et al. } \\
\text { (2007) }\end{array}$ & \\
\hline Statistical mechanics & Poupon et al. (2001), Kreher et al. (2008) & \\
\hline
\end{tabular}

trajectory always tangent to the local vector; it creates a streamline. The very first tractography algorithms published were of that kind (Conturo et al., 1999; Mori et al., 1999; Wedeen, 1996), but very quickly as people started to understand the intrinsic limitation of DTI, they realized that such a simple solution was the source of very obvious artifacts. Researchers began to be aware of the "fibercrossing problem". While a part of the scientific community sought to overcome that problem with new diffusion imaging paradigms, others started to develop cleverer tractography methods that could somehow handle crossings, despite not being effectively mapped by DTI. This generated a very large diversity of methods, having each their advantages and drawbacks. An attempt to classify this non-exhaustive list of solutions is given in Table 1. These attempts were more or less successful since most of the time the price to pay for a better mapping of fiber-crossing in DTI came with more uncertainty and fuzziness in the tracking solutions.

\subsubsection{Challenges and perspectives}

With time it seems that single- and multi-shell HARDI techniques are overtaking DTI-related techniques even those employing the most advanced tractography method. With the advent of HARDI, the fiber-crossing problem has become more tractable, and the field has made a significant step forward. Nevertheless spatial and angular resolution issues remain, and the crossing problem has been shifted into a kiss-crossing problem, as it appears that diffusion profiles of crossing fiber bundles are not distinguishable from so-called kissing fibers. Although this configuration is likely to be less widespread than a crossing bundle, fiber-kissing situations as well as areas of a mixture of kissing and crossing do occur in the brain. This problem again led to investigation of different algorithms in the HARDI setting (Behrens et al., 2007; Gigandet, 2009; Hagmann et al., 2007; Parker and Alexander, 2005; Perrin et al., 2005; Wedeen et al., 2008). Some groups have tried to relate some complex diffusion PDF shapes to either crossing or kissing fiber profiles (Alexander, 2008).

\subsection{From tracts to networks}

\subsubsection{Past and current state of the art}

Understanding the structural basis of functional connectivity patterns requires a comprehensive map of structural connection of the human brain (the human connectome (Hagmann, 2005; Sporns et al., 2005)). We have just seen that advances in diffusion imaging and tractography methods permit the non-invasive mapping of white matter cortico-cortical and cortico-subcortical projections at high spatial resolution. However the path from mapping fiber bundle trajectories to the network representation of the entire brain connectivity requires additional non-trivial processing steps. In the following we briefly survey our approach to the problem.
No single universally accepted parcellation scheme currently exists for human brain regions (e.g. areas of the cerebral cortex), posing a significant challenge to creating a unified resource such as the connectome. Noteworthy is the fact that in the early days of genomics the number and boundaries of genes was not clear either and that actually genomics technology helped to define them (Fields et al., 1994). In the human cerebral cortex, neurons are arranged in an unknown number of anatomically distinct regions and areas, perhaps on the order of 100 or more. It is not clear whether cyto-architechtonically defined (Brodmann, 1985) or more functionally defined areas would be ideal. Nor is it clear either what the optimal scale is for efficient characterization of brain connectivity. Is it the neuronal, micro-column or the regional scale (respectively the micro-, meso- or macro-scale) that is most appropriate?

Since regional delineation is in itself a complex challenge (Schneider, 2009), we simply chose to partition the cortex into small homogenous, compact patches, about $1.5 \mathrm{~cm}^{2}$ in surface area, that are identified according to their gyral coordinates. We postulated that, across subjects, there should be preservation of connectional architecture with respect to gyral geometry. We developed a processing pipeline that is summarized in Fig. 2 and can be briefly described as follows. From diffusion MRI to a highresolution structural connection matrix we adopted a five step process: (1) DSI and high-resolution T1-weighted MRI acquisition of the brain, (2) segmentation of white and gray matter, (3) white matter tractography, (4) segmentation of the cortex into anatomical regions and subdivision into small Regions Of Interest (ROIs) and (5) network/connection matrix construction by computing the fiber connections linking any pair of ROIs.

To segment the cortex, we modified a freely available software (www.freesurfer.org) for partitioning of the cortex into about 1000 homogenous ROIs of about $1.5 \mathrm{~cm}^{2}$, that are in register across subjects such that the nodes of the network, defined by the ROIs, and the connections linking them can be compared and averaged between subjects. Other groups have used similar strategies to partition the cortex using different matching criteria between subjects, using volumetric registration of atlases, like MNI, resulting in smaller networks (Gong et al., 2009; Iturria-Medina et al., 2007; Li et al., 2009). In our early works we used Tailarach coordinates (Hagmann, 2005) or arbitrary subdivisions (Hagmann et al., 2007). In our experience, homogeneity in size and shape of ROIs as well precise anatomical localization is critical in many respects for quantitative analysis of weighted networks.

\subsubsection{Challenges and perspectives}

Important developments are still needed in this processing step of transforming tractographic data into an abstract connectional network. Choosing the right criteria to define an identical node 


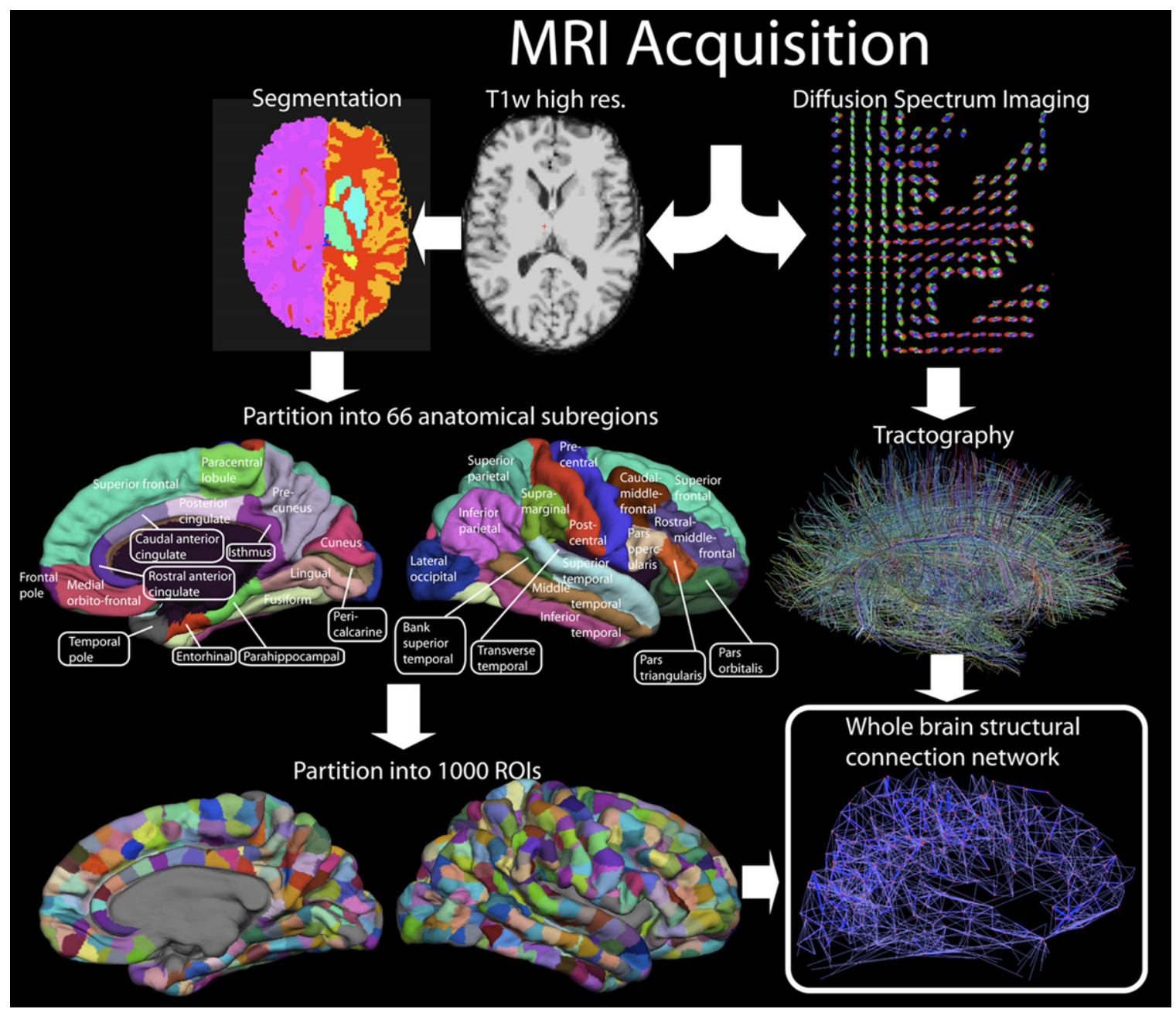

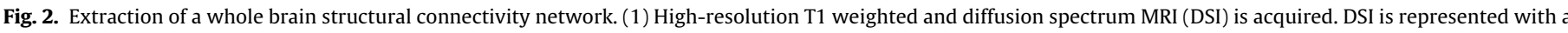

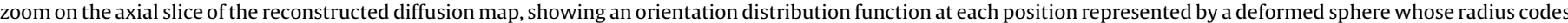

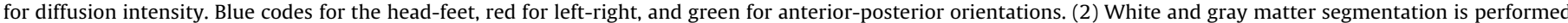

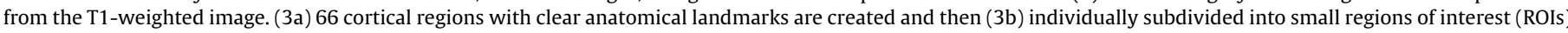

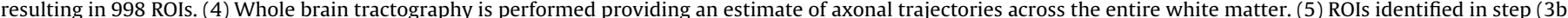

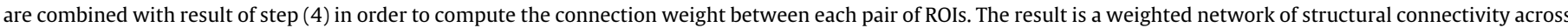
the entire brain. Adapted from PLoS Biology (Hagmann et al., 2008).

between subjects is the first major challenge in our opinion. Should nodes (i.e. ROIs) be labeled according to their precise topological position on the cortical surface (as we have chosen to do), or should they be labeled according to an elaborate fMRI-based functional localization protocol? Should connectivity itself be used to label a node, using preservation of connectional profile as a criterion or should we use a combination of those techniques? At this point the question remains open. Second, what definition of connectivity is going to be most useful? Is the computational connection density (some sort of normalized tractography fiber counting scheme) truly reflective of the underling number of axonal connection per unit surface? What physiological property does mean diffusivity or fractional anisotropy along a tract reflect, and which diffusion-derived parameter best reflects inter-regional connective efficacy?

\subsection{Validation of MR tractography and connectivity maps}

The diversity of diffusion imaging technology and tractography methods naturally brings us to the question of how to benchmark these tools and how to quantitatively assess their performances? The fundamental difference between brain connectional anatomy and the anatomy of the rest of the body is that brain connectivity is largely unknown and that we have no real definite gold standard to refer to. Therefore our ability to evaluate the quality of the tracing just by comparing the shape of reconstructed tracts with our a-priori knowledge is only limited to a crude analysis of a dozen major fiber bundles (Hagmann et al., 2003; Mori et al., 2002). The approach consisting of creating synthetic diffusion models for validation is useful in the development phase to characterize the behavior of a given algorithm, but in our view not adequate to predict the performance in biological tissue (Alexander, 2008; Assaf and Basser, 2005; Hwang et al., 2003; Szafer et al., 1995). Chemical tracing certainly has an important role to play in animal models where some species have been explored relatively extensively-though incompletely and not homogenously (Schmahmann and Pandya, 2006). The macaque is a notable example, where extensive tracing literature is available and has even been collected into a database called CoCoMac (Stephan et al., 2001). Such data is generated from a very heterogeneous material (different individual animals or different species, different techniques) and therefore its consistency and quantification is problematic. Nevertheless it is an extremely valuable resource to compare and validate different techniques. 
(A)

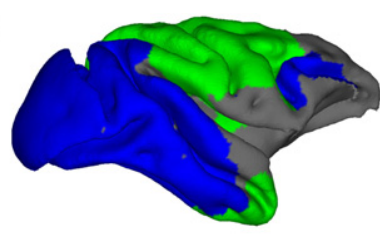

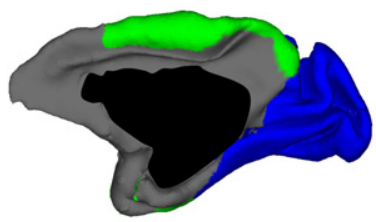

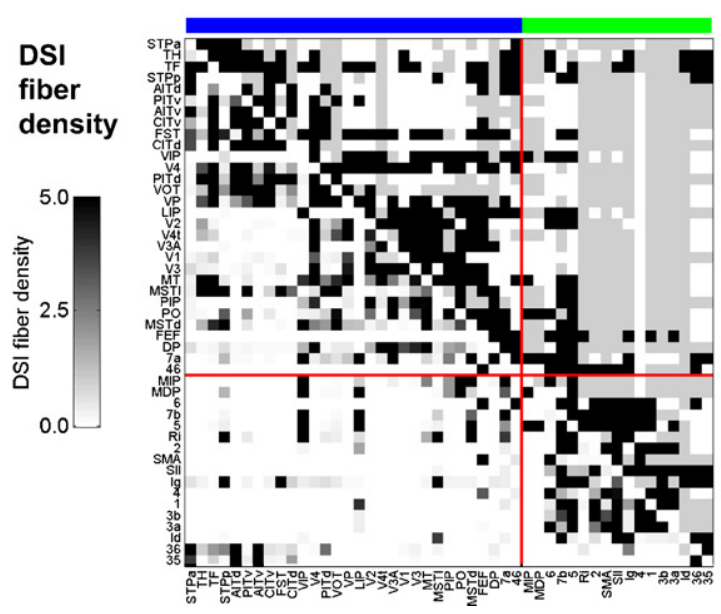

Cocomac data (symmetrized)

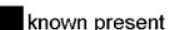

unknown

known absent
(B)

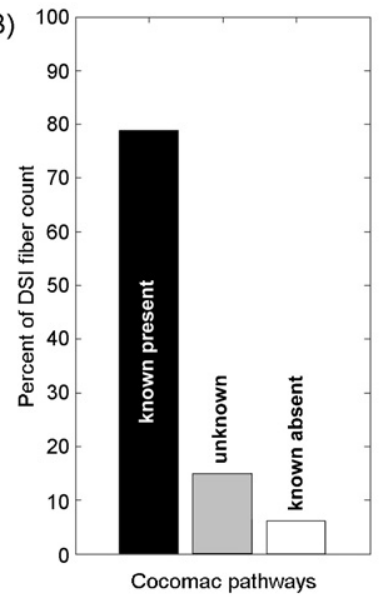

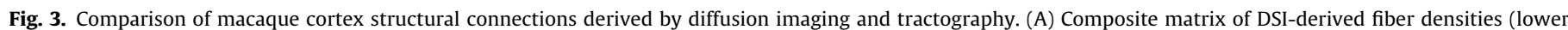

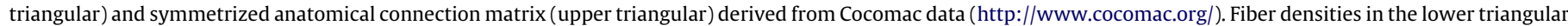

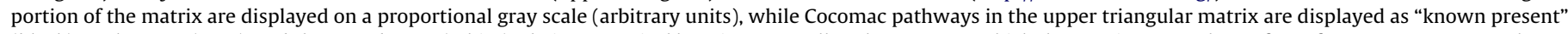

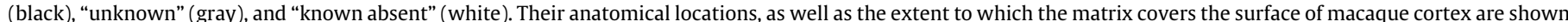

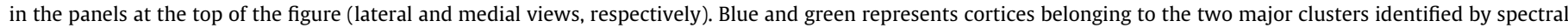

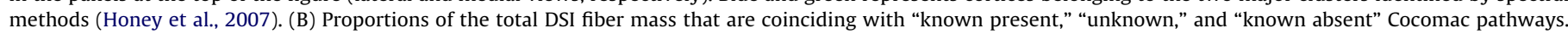

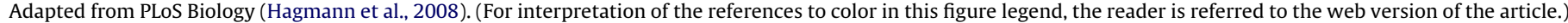

Our group investigated the relation between a macaque brain specimen scanned with DSI and the CoCoMac connectional database. An overlay of structural connectivity derived by these two methods has been studied and is shown in Fig. 3. We found that $78.9 \%$ of all DSI fibers were identified in positions where connections had been identified by tract tracing methods and recorded in Cocomac. A further $15.0 \%$ were placed in positions where the presence or absence of a pathway is currently unknown according to CoCoMac. The remaining $6.1 \%$ were placed in positions where connections had been reported to be absent (Hagmann et al., 2008). The false positive rate was not evaluated in this study.

Using the porcine brain Dyrby et al. (2007) quantitatively and qualitatively assessed the anatomical validity and reproducibility of in vitro multi-fiber probabilistic tractography against two invasive tracers. They demonstrated that probabilistic tractography reliably detected specific pathways and concluded that tractography can be a precise tool in studying anatomical brain connectivity.

In the human brain, chemical tracing data is almost inexistent and limited to case reports (Clarke et al., 1999; Di Virgilio et al., 1999; Tardif and Clarke, 2001) and macroscopic dissection is notoriously unreliable. On the other hand resting-state fMRI has gained in acceptance over the last years for the mapping of functional networks (Raichle et al., 2001; Vincent et al., 2007) that must to some extent be related to the underlying structural connectivity. It has the double advantage of being completely unrelated to diffusion imaging and of being measured in the same scan session and in the same subject.

Fig. $4 \mathrm{~A}$ shows a correlation map of the precuneus and posterior cingulate cortex with the rest of the brain in a resting-state fMRI experiment. These postero-medial structures were previously used for mapping the default mode network (Raichle et al., 2001). In Fig. 4B presents a scatter plot of structural connections derived from DSI and functional connections derived from fMRI for the pre- cuneus and the posterior cingulate cortex over 5 subjects and both hemispheres (Hagmann et al., 2008). The plot indicates that the strengths of structural connections (SC) as estimated from diffusion imaging are highly predictive of the strengths of resting state functional connections in the default mode network ( $\mathrm{rsFC})\left(r^{2}=0.53\right.$, $p<10^{-10}$ ). Scatter plots of structural connections and functional connections for all anatomical subregions averaged over all five participants (Fig. 4C) also revealed significant correlations between their strengths $\left(r^{2}=0.62, p<10^{-10}\right)$. Stronger DSI connections are quantitatively predictive of stronger functional connectivity. The results from this comparison of structural and functional connections not only support the validity of the DSI-derived structural connection patterns but also provides compelling evidence that functional connectivity is reflective, at least in part, of interactions between distant neuronal populations. However, because anatomically unconnected edges exhibit a wide range of rsFC values, it is not possible to reliably infer SC from rsFC using a simple thresholding procedure. The unreliability of inferring SC from the presence of high rsFC results from two factors. First, rsFC can result from mechanisms other than direct SC. These mechanisms include indirect SC, travelling waves in the cortex, and shared cortical innervation from a common source. Second, the base rate of direct SC between two randomly selected $1.5 \mathrm{~cm}^{2}$ patches is very low. This difficulty in inferring SC from rsFC is not just a reflection of the practical limitations of fMRI: a similar unreliability was also seen in our computational model results - in which SC provided the exact coupling matrix and there was no "acquisition noise" (Honey et al., 2009). We note that, although the relationship between indirect SC and rsFC is weaker than the relationship between direct SC and $\mathrm{rsFC}$, it is nonetheless highly significant. Consistent with the observation of Vincent et al. (2007), that interhemispheric rsFC between the visual cortices is most likely subserved by polysynaptic connections, we find in our data that indirect cortico-cortical SC is an especially strong predictor of 
(A)
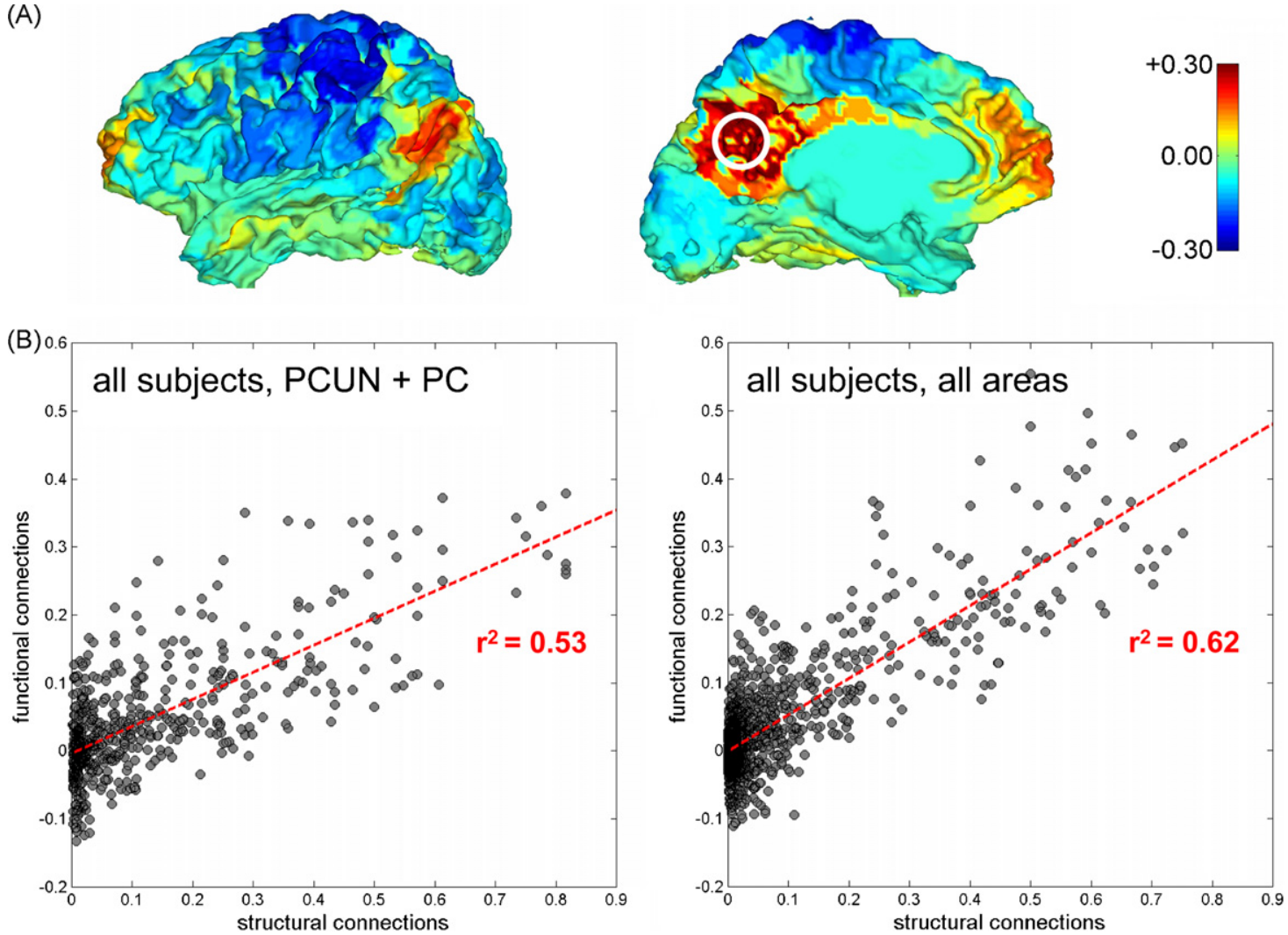

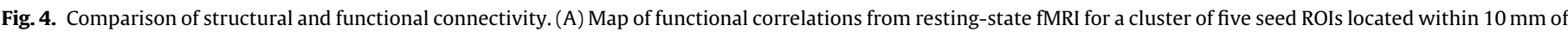

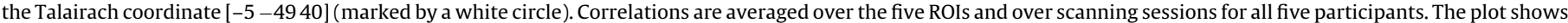

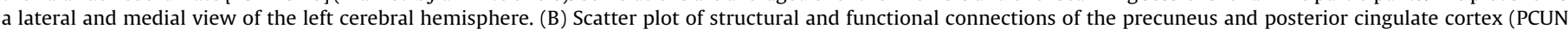

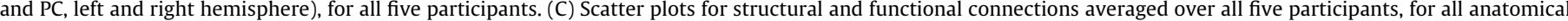
subregions in both hemispheres. Adapted from PLoS Biology (Hagmann et al., 2008).

the rsFC between the visual cortices of each hemisphere (Honey et al., 2009).

Obviously, extensive and large-scale post-mortem macaque and in vivo human studies are urgently needed in order to appropriately validate diffusion MRI tractography and in order to be able to set clear sensitivity and specificity boundaries for MR tractography.

\section{Analyzing the connectome}

\subsection{Brain structural network analysis}

Once an abstract network has been defined, sophisticated networks analysis can be deployed to determine key aspects of the brain's structural organization. These methods have been widely applied across multiple areas of science (Strogatz, 2001), including networks of interacting proteins, ecosystems, communication and transportation, the world wide web, social systems, and finally, the brain (Bullmore and Sporns, 2009). Most relevant for neuroscience and the connectome are networks measures that evaluate the degree of clustering and modularity (measures of functional segregation), and of path length and communication efficiency (measures of functional integration). In a seminal study (Watts and Strogatz, 1998) discovered that networks combining high clustering of local connections and a short path length due to a small fraction of long-range connections were organized as a "smallworld". In such networks, any node can be reached from any other node in a small number of steps, despite the fact that most nodes form tightly coupled local communities. Hence such networks combine functional integration and functional segregation.
A number of studies have since shown that large-scale anatomical networks of the mammalian cerebral cortex exhibit small-world attributes (Bassett and Bullmore, 2006). Small-world structures has been reported to exist in the cortex of non-human primates based on data derived by classical tract tracing (Sporns et al., 2000; Sporns and Zwi, 2004), as well as in networks derived from diffusion imaging of the human brain (Gong et al., 2009; Hagmann et al., 2008, 2007; Iturria-Medina et al., 2008), see Fig. 5. The detection of small-world attributes appears to be largely independent of the parcellation scheme or the spatial resolution and is therefore a robust indicator of the existence of local communities or modules that are globally interconnected. The brain regions that link together local communities have special functional roles in maintaining the overall integrity of the network, as well as possibly controlling patterns of information flow between modules. A set of network measures originally defined in the social sciences can assess the degree of influence of individual regions or connections on the flow of information in the network. These measures of "centrality" assess how many of the network's shortest paths travel through each region or connection. Regions of high centrality participate in many of these short paths and are often essential for linking different communities to each other. Their loss is particularly disruptive to the network (Honey and Sporns, 2008). In the human brain, several regions in the frontal and parietal cortex have high centrality, particularly the posterior cingulate and precuneus (Hagmann et al., 2008), a key region of the brain's default mode network, see Figs. 5 and 6.

Network analysis tools are likely to gain importance as more connection data sets from healthy human participants and from people with clinical conditions become available. Network metrics 

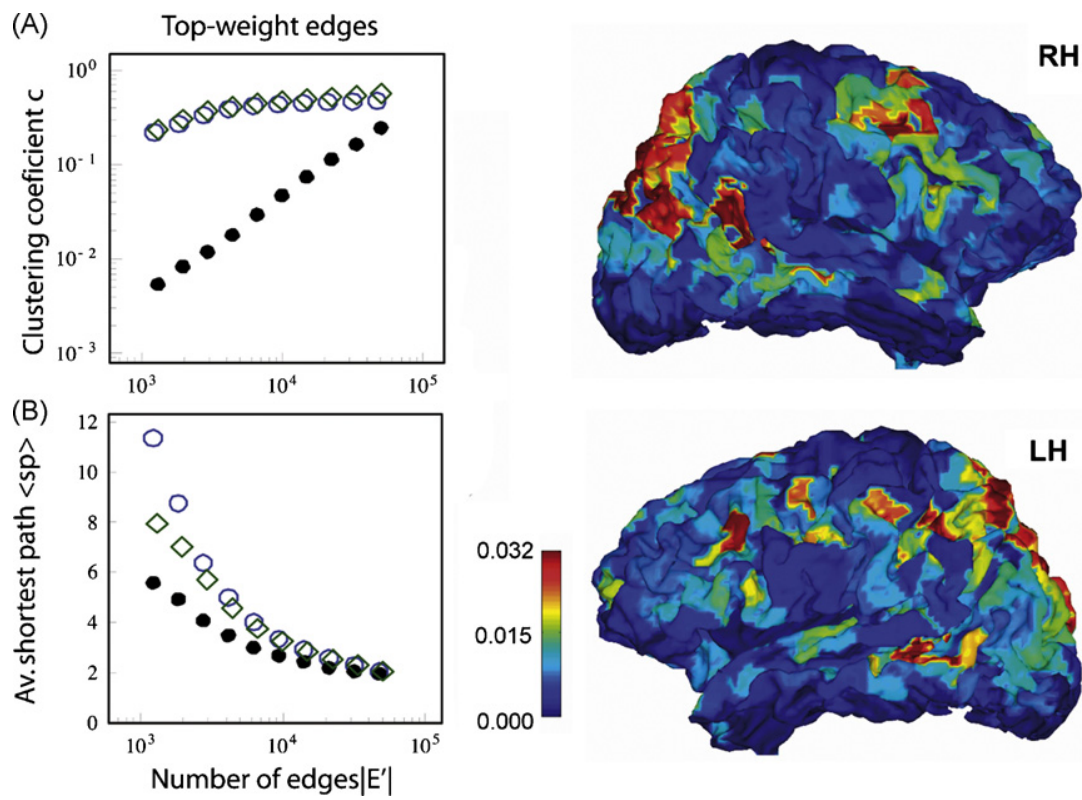

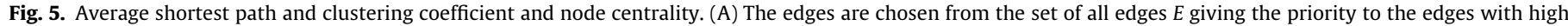

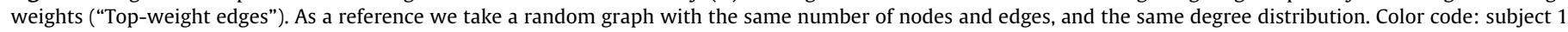

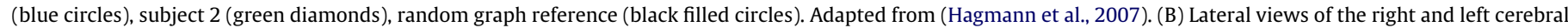
hemispheres showing ROI centrality, averaged across five. Adapted from PLoS Biology (Hagmann et al., 2008).

are beginning to be applied in comparison studies of the structural connectivity of healthy and diseased brains (e.g. Bassett et al., 2008), and they may become important diagnostic tools in future clinical applications of the connectome.

\subsection{Functional connectomics}

A major motivation for the comprehensive mapping of connectivity in mammalian brains is the possibility that such maps will

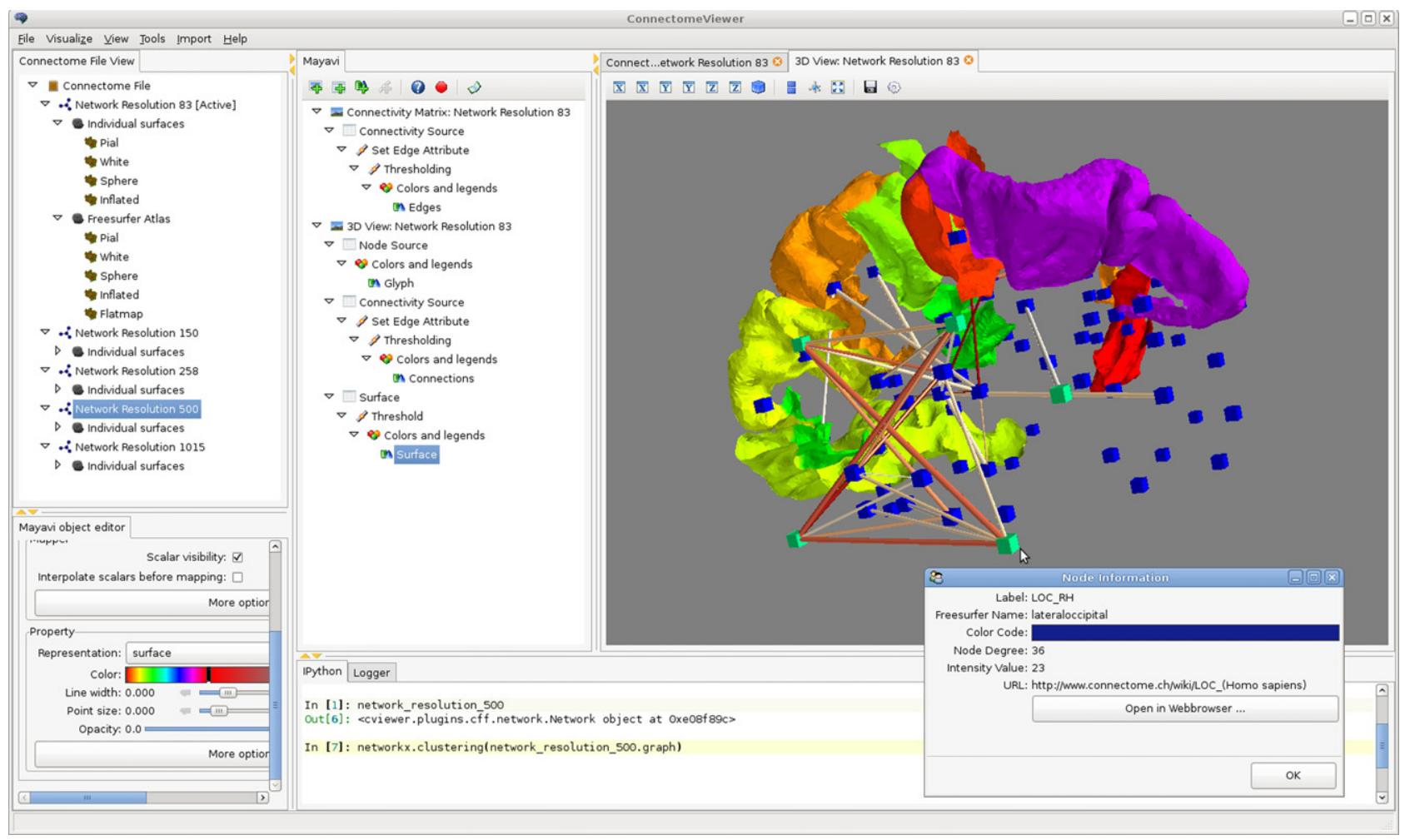

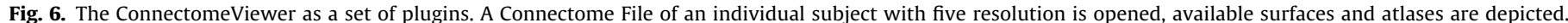

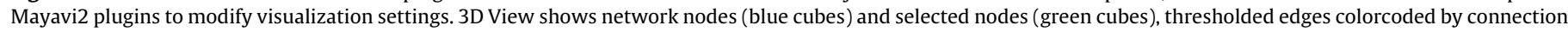

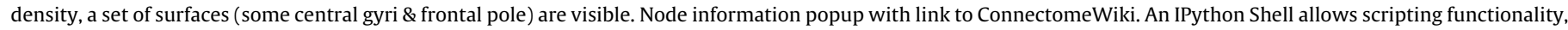
the command to compute clustering of the Resolution 500 network is shown. 
provide a window onto the large-scale functional architecture of the mammalian brain. If human structural connection networks can be reliably partitioned into tens or hundreds of distinct network modules, then it is expected that these modules will correspond to functionally localized subdivisions of the brain. Indeed, connectionbased subdivisions of premotor cortex (Johansen-Berg et al., 2004) have been found to correspond closely to the subdivisions identified using functional imaging of motor and linguistic tasks in the same individuals, and connection-based subdivisions of thalamus (Zhang et al., 2008) as well as inferior frontal cortex (Anwander et al., 2007) and cingulate cortices (Beckmann et al., 2009) have been found to match the canonical functional parcellations of these areas. In a less function-specific vein, other groups have investigated the relationship, across the brain, between so-called "functional connectivity" which is assessed using correlations in resting-state BOLD signal with structural connectivity identified using diffusion imaging (Greicius et al., 2009; Hagmann et al., 2008; Honey et al., 2009; Koch et al., 2002; Skudlarski et al., 2008; van den Heuvel et al., 2008, 2009; van den Heuvel et al., 2009; Van Dijk et al., 2010). These studies are of a more exploratory nature, because the functional significance and neurophysiological basis of the correlation patterns in resting-state BOLD signal are not well understood. On the other hand, these studies promise to aid our understanding of distributed and general organizational processes (Fox and Raichle, 2007) in the mammalian brain, as well as their large-scale structural underpinning.

Ultimately, one would like to go beyond the observation of a simple correlation between structural integrity and mean population-level neuronal activity. Towards this objective, a number of computational studies have attempted to combine interregional connectivity data with intra-regional circuit and cellular properties to produce large-scale models of cortical (Ghosh et al., 2008; Honey et al., 2007, 2009; Knock et al., 2009) and corticothalamic function (Izhikevich and Edelman, 2008). The value of modeling studies is twofold. First, models produce predictions of the kind and the strength of interactions that should be empirically observed between macroscopic assemblies (such as ROIs) on the basis of the interactions occurring between their microscopic constituents (i.e. neurons and neuronal assemblies). Second, models are important in helping us to understand how higher-order network features of the anatomical structure (e.g. locally high centrality, or modularity) will manifest in the functional networks observed in neuroimaging experiments.

Although large-scale computational models are at present only rough approximations of the complex multi-scale dynamics that actually occur within the brain, they provide a crucial connection between diffusion MRI studies and the larger body of neuroscientific and clinical research, and an important tool for exploring the relationship between large-scale neural structure and function.

\section{Looking towards the future of connectomic research}

As we mentioned earlier, there is still significant efforts that need to be put in the improvement of the technology dedicated to the acquisition of connectomic data. Better MRI technology, better tractography and registration methods will be developed and will improve resolution, reliability and artifacts. In addition efforts will be made to ease analysis and organization of such data and very diverse biological questions may potentially find an answer with connectomic research.

\subsection{Missing tools}

As we have just seen, large-scale connectional networks directly derived from in vivo imaging are getting more readily available and important questions can be answered using network visualization and analysis approaches. Generic tools already exist to analyze and visualize large-scale networks like Pajek (Batagelj and Mrvar, 2003) or NetworkX (networkx.lanl.gov). Those and many others where mainly used and developed for telecom (GraphViz, www.graphviz.org) and theoretical social sciences (SocSciBot Network, webometrics.wlv.ac.uk/networkhelp). Since they are not dedicated to neuro-science, their use can be quite cumbersome in this setting. Packages specifically designed for brain network analysis begin to emerge and are currently being developed in order to meet this growing need to apply powerful network measure and visualization techniques to neuro-networks. An example is the brain connectivity toolbox (Rubinov and Sporns, 2009) and our the ConnectomeViewer (Gerhard et al., 2009). Through the development of this viewer, we are providing the scientific community with an extensible tool for visualization and analysis of connectomic data. A dedicated file format is defined; brain networks are visualized in the form of directed and undirected graphs embedded in 3D. Different layouts can be used. The python large-scale network environment, NetworkX, is embedded and can be used to perform analysis. Progressively dedicated useful brain network analysis plugins will be added and hopefully will meet the community's needs.

Not only network measures and visualization are important for connectomic research but also systematic data organization and sharing is essential. Visionary work has been done by Rolf Kötter and his colleagues several years ago for the Macaque (Stephan et al., 2001). They developed an open database collecting and organizing a very large amount of Macaque tracing studies (www.cocomac.org). This facilitated immensely the emergence of pioneering studies on brain network topology (e.g. Sporns and Kotter, 2004). An other trans-species data-basing effort readily available is "The Brain Architecture Management System (BAMS)" (Bota et al., 2003). Recently, international efforts have begun to build standardized ontologies in neuro-science (www.neurolex.org) especially important in the field of neuroanatomy (Workshop on Neuroanatomical Nomenclature and Taxonomy, September 10-11, 2007, www.incf.org). We believe that embedding connectomics research in this landscape, in order to provide highly standardized and widely available connectional information for the human brain would be very important to the entire neuro-science community. Much like the existing brain databases such as Cocomac or BAMS we plan to develop a fully integrated connectome wiki and database that would provide the means to store and query easily the large datasets coming from MRI connectomics in the upcoming years (www.connectomics.org).

\subsection{Neuro-science questions that may benefit from connectomics}

Structural and functional imaging developments combined will provide us with a more and more comprehensive map of brain connectivity, which is expected in the near future to be at the root of a significant improvement in our understanding how our brain works in the healthy and pathologic state, as well as in the course of development and senescence.

Large studies will be needed that combine on one hand, high quality large structural and functional connectomic imaging data and on the other hand collect elaborate demographic, psychosomato-motor, psychosocial and cognitive data as well as genomic data. The powerful combination of this information and connectome analysis should begin to expose the principles governing brain network topology as well as its normal variation. Building on these governing principles, sophisticated network models can then be developed to further probe the link between structure and function. Not only will the variation of structural and functional connectional patterns be characterized but also interdependencies 
will be studied. The combination of connectomic information with demographic, social, cognitive and somato-motor data will allow us to draw many statistical relationships to probe the complex relationship between the latter and brain organization.

Even more exciting is the potential inferences we will be able to draw from a clever combination of large population-based connectomic and genomic information. Indeed, our knowledge of genetic and epigenetic influences on brain development, including mechanisms governing neuronal migration and connectivity is rapidly growing, as are the genomic databases (Bystron et al., 2008; Jakovcevski et al., 2009; Mochida and Walsh, 2004) (www.ornl.gov/sci/techresources/Human_Genome/home.shtml). Such large-scale genomic data combined with the high density of information provided by a contectome database will be transformative. By combining the information in these databases we can now begin to explore the genomic determinants of neuronal network topology, network myelination (by combining our techniques with diffusivity, magnetization transfer or T1 relaxation measurements (Barnea-Goraly et al., 2005; Gogtay et al., 2004; Xydis et al., 2006)), network information flow and functional interaction (using EEG and MEG).

The ability to characterize and probe the human connectome will have a dramatic impact when focused on not only normal adults but also the developing, degenerating and diseased brain. From birth to early adulthood the brain undergoes dramatic modifications, with neuronal loss, dendritic proliferation and axonal growth followed by reductions in synaptic plasticity, axonal pruning and myelination (Bystron et al., 2008; Innocenti and Price, 2005; Johnston et al., 2009). These processes result in gyral folding, regional specification and ultimately network optimization. Similarly at the other end of age spectrum, normal degenerative mechanisms induce changes in the brain white and gray matter causing neuronal loss and modification of neuronal information transport efficacy which inevitably translate themselves as cognitive decline (Damoiseaux et al., 2008; Meunier et al., 2009). The genetic and epigenetic influences on network topology and the implications for cognitive function across the trajectory of development and decline are just beginning to be unraveled.

In addition the neuro-science community is becoming increasingly aware that mis- or dis-connectivity is implicated not only in the etiology of many neuro-psychiatric diseases but also as the primary mechanism for observed morphometric changes (Kyriakopoulos et al., 2008; Shenton et al., 2001; Thompson and Apostolova, 2007). Schizophrenia and Alzheimer's disease are the two most prominent examples (Bassett et al., 2008; Buckner et al., 2009). In both cases it has been shown that widespread changes in cortical thickness, white matter structure, brain electrophysiology and metabolism occur and are hypothesized to be related to changes in the underlying connectivity, which likely precisely translates into the related cognitive impairment. Similarly in developmental disorders such as autism, theories of mis- and disconnection are emerging with the view that in autism small-world connections dominate with deficits in control and integration and control governed by long-range connections (Casanova and Trippe, 2009; Frith, 2004; Geschwind and Levitt, 2007; Perez Velazquez et al., 2009).

\section{Conclusion}

In vivo and post-mortem diffusion MRI advances, in combination with sophisticated post-processing methods, are opening a new field called MR connectomics. This field is not solely about developing technique for high-throughput and high-resolution connectional mapping. In combination with functional techniques, it is more importantly the field that will provide us with the right framework to consider the brain's complexity.

\section{Acknowledgments}

This work is supported by the Swiss National Science Foundation, the Center for Biomedical Imaging (CIBM) of the Geneva Lausanne Universities and the EPFL, and the foundations Leenaards and Louis-Jeantet and the JS McDonnell Foundation.

\section{References}

Alexander DC. A general framework for experiment design in diffusion MRI and its application in measuring direct tissue-microstructure features. Magn Reson Med 2008;60:439-48.

Anwander A, Tittgemeyer M, von Cramon DY, Friederici AD, Knosche TR. Connectivity-based parcellation of Broca's area. Cereb Cortex 2007;17:816-25.

Assaf Y, Basser PJ. Composite hindered and restricted model of diffusion (CHARMED) MR imaging of the human brain. Neuroimage 2005;27:48-58.

Barnea-Goraly N, Menon V, Eckert M, Tamm L, Bammer R, Karchemskiy A, et al. White matter development during childhood and adolescence: a cross-sectional diffusion tensor imaging study. Cereb Cortex 2005;15:1848-54

Basser PJ, Mattiello J, LeBihan D. MR diffusion tensor spectroscopy and imaging. Biophys J 1994;66:259-67.

Basser PJ, Pajevic S, Pierpaoli C, Duda J, Aldroubi A. In vivo fiber tractography using DT-MRata. Magn Reson Med 2000;44(4):625-32.

Bassett DS, Bullmore E. Small-world brain networks. Neuroscientist 2006;12:512-23.

Bassett DS, Bullmore E, Verchinski BA, Mattay VS, Weinberger DR, MeyerLindenberg A. Hierarchical organization of human cortical networks in health and schizophrenia. J Neurosci 2008;28:9239-48.

Batagelj V, Mrvar A. Pajek-analysis and visualization of large networks. In: Jünger M, Mutzel P, editors. Graph drawing software. Berlin: Springer; 2003. p. 77-103.

Beaulieu C. The basis of anisotropic water diffusion in the nervous system-a technical review. NMR Biomed 2002;15:435-55.

Beckmann M, Johansen-Berg H, Rushworth MF. Connectivity-based parcellation of human cingulate cortex and its relation to functional specialization. J Neurosci 2009;29:1175-90.

Behrens TE, Woolrich MW, Jenkinson M, Johansen-Berg H, Nunes RG, Clare S, Matthews PM, Brady JM, Smith SM. Characterization and propagation of uncertainty in diffusion-weighted MR imaging. Magn Reson Med 2003;50(5):1077-88.

Behrens TE, Berg HJ, Jbabdi S, Rushworth MF, Woolrich MW. Probabilistic diffusion tractography with multiple fibre orientations: what can we gain? Neuroimage 2007;34:144-55.

Bohland JW, Wu C, Barbas H, Bokil H, Bota M, Breiter HC, et al. A proposal for a coordinated effort for the determination of brainwide neuroanatomical connectivity in model organisms at a mesoscopic scale. PLoS Comput Biol 2009;5:e1000334.

Bota M, Dong HW, Swanson LW. From gene networks to brain networks. Nat Neurosci 2003;6:795-9.

Brodmann K. Vergleichende Lokalisationslehre der Grosshirnrinde in ihren Prinzipien dargestellt auf Grund des Zellenbaues, Reprint der Originalausgabe von 1909, (1. Aufl.)/mit einem Nachwort und einem Literaturverzeichnis von Ernst Winkelmann, Leipzig, und Karl Seidel, Berlin ed. Barth: Leipzig, 1985.

Buckner RL, Sepulcre J, Talukdar T, Krienen FM, Liu H, Hedden T, et al. Cortical hubs revealed by intrinsic functional connectivity: mapping, assessment of stability, and relation to Alzheimer's disease. J Neurosci 2009;29:1860-73.

Bullmore E, Sporns O. Complex brain networks: graph theoretical analysis of structural and functional systems. Nature Rev 2009;10:186-98.

Bystron I, Blakemore C, Rakic P. Development of the human cerebral cortex: Boulder Committee revisited. Nature Rev 2008;9:110-22.

Callaghan PT. Principles of nuclear magnetic resonance microscopy. Oxford: Clarendon Press; 1991.

Casanova M, Trippe J. Radial cytoarchitecture and patterns of cortical connectivity in autism. Phil Trans Royal Soc Lond 2009;364:1433-6.

Clarke S, Riahi-Arya S, Tardif E, Eskenasy AC, Probst A. Thalamic projections of the fusiform gyrus in man. Eur J Neurosci 1999;11:1835-8.

Conturo TE, Lori NF, Cull TS, Akbudak E, Snyder AZ, Shimony JS, et al. Tracking neuronal fiber pathways in the living human brain. Proc Natl Acad Sci USA 1999;96:10422-7.

Cory D, Garroway A. Measurements of translational displacement probabilities by nmr-an indicator of compartmentation. Magn Res Med 1990;14:435-44.

Crick F, Jones E. Backwardness of human neuroanatomy. Nature 1993;361:109-10.

Damoiseaux JS, Beckmann CF, Arigita EJ, Barkhof F, Scheltens P, Stam CJ, et al. Reduced resting-state brain activity in the "default network" in normal aging. Cereb Cortex 2008;18:1856-64.

Di Virgilio G, Clarke S, Pizzolato G, Schaffner T. Cortical regions contributing to the anterior commissure in man. Exp Brain Res 1999;124:1-7.

Douek P, Turner R, Pekar J, Patronas N, Le Bihan D. MR color mapping of myelin fiber orientation. J Comput Assist Tomogr 1991;15:923-9. 
Dyrby TB, Sogaard LV, Parker GJ, Alexander DC, Lind NM, Baare WF, et al. Validation of in vitro probabilistic tractography. Neuroimage 2007;37:1267-77.

Einstein A. Investigations on the theory of the Brownian movement. Dover Publications; 1956.

Fields CAM, White O, Venter JC. How many genes in the human genome? Nat Genet 1994; 7:345-6.

Fox MD, Raichle ME. Spontaneous fluctuations in brain activity observed with functional magnetic resonance imaging. Nature Rev 2007;8:700-11.

Frith C. Is autism a disconnection disorder? Lancet Neurol 2004;3:577.

Gerhard S, Hagmann P, Cammoun L, Gigandet X, Thiran J-P, Meuli R. Connectome Viewer; 2009. www.connectomics.org.

Geschwind DH, Levitt P. Autism spectrum disorders: developmental disconnection syndromes. Curr Opin Neurobiol 2007;17:103-11.

Ghosh A, Rho Y, McIntosh AR, Kotter R, Jirsa VK. Noise during rest enables the exploration of the brain's dynamic repertoire. PLoS Comput Biol 2008;4:e1000196.

Gigandet X. Global brain connectivity analysis by diffusion MR tractography: algorithms, validation and applications. PhD thesis at Signal Processing Laboratory 5. Ecole Polytechnique Fédérale de Lausanne (EPFL): Lausanne; 2009.

Gogtay N, Giedd J, Lusk L, Hayashi K, Greenstein D, Vaituzis A, et al. Dynamic mapping of human cortical development during childhood through early adulthood. Proc Natl Acad Sci USA 2004;101:8174-9.

Gong G, He Y, Concha L, Lebel C, Gross DW, Evans AC, Beaulieu C. Mapping anatomical connectivity patterns of human cerebral cortex using in vivo diffusion tensor imaging tractography. Cereb Cortex 2009;19(3):524-36, Epub 2008 Jun 20.

Greicius MD, Supekar K, Menon V, Dougherty RF. Resting-state functional connectivity reflects structural connectivity in the default mode network. Cereb Cortex 2009;19:72-8.

Gresham D, Dunham MJ, Botstein D. Comparing whole genomes using DNA microarrays. Nat Rev Genet 2008;9:291-302.

Hagmann P. From diffusion MRI to brain connectomics. Lausanne: Signal Processing Institute. Ecole Polytechnique Fédérale de Lausanne (EPFL); 2005. p. 127.

Hagmann P, Cammoun L, Gigandet X, Meuli R, Honey CJ, Wedeen VJ, et al. Mapping the structural core of human cerebral cortex. PLoS Biol 2008;6: e159.

Hagmann P, Jonasson L, Maeder P, Thiran JP, Wedeen VJ, Meuli R. Understanding diffusion MR imaging techniques: from scalar diffusion-weighted imaging to diffusion tensor imaging and beyond. Radiographics 2006;26(Suppl 1): S205-23.

Hagmann P, Kurant M, Gigandet X, Thiran P, Wedeen VJ, Meuli R, et al. Mapping human whole-brain structural networks with diffusion MRI. PLoS ONE 2007;2:e597.

Hagmann P, Thiran JP, Jonasson L, Vandergheynst P, Clarke S, Maeder P, et al. DTI mapping of human brain connectivity: statistical fibre tracking and virtual dissection. Neuroimage 2003;19:545-54.

Honey CJ, Kotter R, Breakspear M, Sporns O. Network structure of cerebral cortex shapes functional connectivity on multiple time scales. Proc Natl Acad Sci USA 2007;104:10240-5.

Honey CJ, Sporns O. Dynamical consequences of lesions in cortical networks. Human Brain Mapp 2008;29:802-9.

Honey CJ, Sporns O, Cammoun L, Gigandet X, Thiran JP, Meuli R, et al. Predicting human resting-state functional connectivity from structural connectivity. Proc Natl Acad Sci USA 2009;106:2035-40.

Hutchison 3rd CA. DNA sequencing: bench to bedside and beyond. Nucleic Acids Res 2007;35:6227-37.

Hwang SN, Chin CL, Wehrli FW, Hackney DB. An image-based finite difference model for simulating restricted diffusion. Magn Reson Med 2003;50:373-82.

Innocenti GM, Price DJ. Exuberance in the development of cortical networks. Nature Rev 2005;6:955-65.

Iturria-Medina Y, Canales-Rodriguez EJ, Melie-Garcia L, Valdes-Hernandez PA, Martinez-Montes E, Aleman-Gomez Y, et al. Characterizing brain anatomical connections using diffusion weighted MRI and graph theory. Neuroimage 2007;36:645-60.

Iturria-Medina Y, Sotero RC, Canales-Rodriguez EJ, Aleman-Gomez Y, Melie-Garcia L. Studying the human brain anatomical network via diffusion-weighted MRI and Graph Theory. Neuroimage 2008;40:1064-76.

Izhikevich EM, Edelman GM. Large-scale model of mammalian thalamocortical systems. Proc Natl Acad Sci USA 2008;105:3593-8.

Jakovcevski I, Filipovic R, Mo Z, Rakic S, Zecevic N. Oligodendrocyte development and the onset of myelination in the human fetal brain. Front Neuroanat 2009; $3: 5$.

Jansons K, Alexander D. Persistent angular structure: new insights from diffusion magnetic resonance imaging data. Inverse Probl 2003;19:1031-46.

Jbabdi S, Woolrich MW, Andersson JL, Behrens TE. A Bayesian framework for global tractography. Neuroimage 2007;37(1):116-29, Epub 2007 Apr 27.

Johansen-Berg H, Behrens TE, Robson MD, Drobnjak I, Rushworth MF, Brady JM, et al. Changes in connectivity profiles define functionally distinct regions in human medial frontal cortex. Proc Natl Acad Sci USA 2004;101:13335-40.

Johnston MV, Ishida A, Ishida WN, Matsushita HB, Nishimura A, Tsuji M. Plasticity and injury in the developing brain. Brain Dev 2009;31:1-10.

Knock SA, McIntosh AR, Sporns O, Kötter R, Hagmann P, Jirsa VK. The effects of physiologically plausible connectivity structure on local and global dynamics in large scale brain models. J Neurosci Methods 2009;183(1):86-94, Epub 2009 Jul 14.

Koch MA, Norris DG, Hund-Georgiadis M. An investigation of functional and anatomical connectivity using magnetic resonance imaging. Neuroimage 2002;16:241-50.
Kreher BW, Mader I, Kiselev VG. Gibbs tracking: a novel approach for the reconstruction of neuronal pathways. Magn Reson Med 2008;60(4):953-63.

Krueger G, editor. Application guide EP2D_DSI work-in-progress package for diffusion spectrum imaging in siemens; 2008.

Kyriakopoulos M, Bargiotas T, Barker GJ, Frangou S. Diffusion tensor imaging in schizophrenia. Eur Psychiatry 2008;23:255-73.

Lazar M, Weinstein DM, Tsuruda JS, Hasan KM, Arfanakis K, Meyerand ME, Badie B, Rowley HA, Haughton V, Field A, Alexander AL. White matter tractography using diffusion tensor deflection. Hum Brain Mapp 2003;18(4):306-21.

Le Bihan D. Looking into the functional architecture of the brain with diffusion MRI. Nature Rev Neurosci 2003;4:469-80.

Le Bihan D. Molecular diffusion, tissue microdynamics and microstructure. NMR Biomed 1995;8:375-86.

Li Y, Liu Y, Li J, Qin W, Li K, Yu C, et al. Brain anatomical network and intelligence. PLoS Comput Biol 2009;5:e1000395.

Meunier D, Achard S, Morcom A, Bullmore E. Age-related changes in modular organization of human brain functional networks. Neuroimage 2009;44: 715-23.

Mo ML, Palsson BO. Understanding human metabolic physiology: a genome-tosystems approach. Trends Biotechnol 2009;27:37-44.

Mochida GH, Walsh CA. Genetic basis of developmental malformations of the cerebral cortex. Arch Neurol 2004;61:637-40.

Mori S, Crain BJ, Chacko VP, van Zijl PC. Three-dimensional tracking of axonal projections in the brain by magnetic resonance imaging. Annals Neurol 1999;45:265-9.

Mori S, Kaufmann WE, Davatzikos C, Stieltjes B, Amodei L, Fredericksen K, et al. Imaging cortical association tracts in the human brain using diffusion-tensorbased axonal tracking. Magn Reson Med 2002;47:215-23.

Moseley ME, Kucharczyk J, Mintorovitch J, Cohen Y, Kurhanewicz J, Derugin N, et al Diffusion-weighted MR imaging of acute stroke: correlation with T2-weighted and magnetic susceptibility-enhanced MR imaging in cats. Ajnr 1990;11: 423-9.

Parker GJ, Alexander DC. Probabilistic anatomical connectivity derived from the microscopic persistent angular structure of cerebral tissue. Phil Trans Royal Soc Lond 2005;360:893-902.

Parker GJ, Wheeler-Kingshott CA, Barker GJ. Estimating distributed anatomical connectivity using fast marching methods and diffusion tensor imaging. IEEE Trans Med Imaging 2002;21(5):505-12.

Parker GJ, Haroon HA, Wheeler-Kingshott CA. A framework for a streamline-based probabilistic index of connectivity (PICo) using a structural interpretation of MRI diffusion measurements. J Magn Reson Imaging 2003;18(2): 242-54.

Perez Velazquez JL, Barcelo F, Hung Y, Leshchenko Y, Nenadovic V, Belkas J, et al. Decreased brain coordinated activity in autism spectrum disorders during executive tasks: reduced long-range synchronization in the fronto-parietal networks. Int J Psychophysiol 2009;73:341-9.

Perrin M, Poupon C, Cointepas Y, Rieul B, Golestani N, Pallier C, et al. Fiber tracking in q-ball fields using regularized particle trajectories. Inf Process Med Imaging 2005;19:52-63.

Poupon C, Mangin J, Clark CA, Frouin V, Régis J, Le Bihan D, Bloch I. Towards inference of human brain connectivity from MR diffusion tensor data. Med Image Anal 2001;5(1):1-15.

Raichle ME, MacLeod AM, Snyder AZ, Powers WJ, Gusnard DA, Shulman GL. A default mode of brain function. Proc Natl Acad Sci USA 2001;98:676-82.

Reese TG, Benner T, Wang R, Feinberg DA, Wedeen VJ. Halving imaging time of whole brain diffusion spectrum imaging and diffusion tractography using simultaneous image refocusing in EPI. J Magn Reson Imaging 2009;29: $517-22$.

Rubinov M, Sporns O. Complex network measures of brain connectivity: Uses and interpretations. Neuroimage 2009 Oct 9. [Epub ahead of print] http://sites.google.com/a/brain-connectivity-toolbox.net/bct/Home.

Schmahmann JD, Pandya DN. Fiber pathways of the brain. New York: Oxford University Press; 2006

Schneider W. Brain connectivity competition. Pittsburgh; 2009.

Seunarine K, Alexander D. Multiple fibres: beyond the diffusion tensor. In: JohansenBerg H, Behrens T, editors. Diffusion MRI. Elsevier; 2009.

Shenton ME, Dickey CC, Frumin M, McCarley RW. A review of MRI findings in schizophrenia. Schizophr Res 2001;49:1-52.

Skudlarski P, Jagannathan K, Calhoun VD, Hampson M, Skudlarska BA, Pearlson G. Measuring brain connectivity: diffusion tensor imaging validates resting state temporal correlations. Neuroimage 2008;43:554-61.

Sporns O, Kotter R. Motifs in brain networks. PLoS Biol 2004;2:1910-8.

Sporns O, Tononi G, Edelman GM. Theoretical neuroanatomy: relating anatomical and functional connectivity in graphs and cortical connection matrices. Cereb Cortex 2000;10:127-41.

Sporns O, Tononi G, Kotter R. The human connectome: a structural description of the human brain. PLoS Comput Biol 2005;1:e42.

Sporns O, Zwi JD. The small world of the cerebral cortex. Neuroinformatics 2004;2:145-62.

Staempfli P, Jaermann T, Crelier GR, Kollias S, Valavanis A, Boesiger P. Resolving fiber crossing using advanced fast marching tractography based on diffusion tensor imaging. Neuroimage 2006;30(1):110-20, Epub 2005 Oct 24.

Stephan KE, Kamper L, Bozkurt A, Burns GA, Young MP, Kotter R. Advanced database methodology for the Collation of Connectivity data on the Macaque brain ( $\mathrm{CoCo}-$ Mac). Phil Trans Royal Soc Lond 2001;356:1159-86.

Strogatz S. Exploring complex networks. Nature 2001;410:268-76. 
Szafer A, Zhong J, Gore JC. Theoretical model for water diffusion in tissues. Magn Reson Med 1995;33:697-712.

Tardif E, Clarke S. Intrinsic connectivity of human auditory areas: a tracing study with DiI. Eur J Neurosci 2001;13:1045-50.

Thompson PM, Apostolova LG. Computational anatomical methods as applied to ageing and dementia. Br J Radiol 2007;80(Spec No 2):S78-91.

Tournier JD, Calamante F, Gadian DG, Connelly A. Direct estimation of the fiber orientation density function from diffusion-weighted MRI data using spherical deconvolution. Neuroimage 2004;23:1176-85.

Tuch DS, Reese TG, Wiegell MR, Makris N, Belliveau JW, Wedeen VJ. High angular resolution diffusion imaging reveals intravoxel white matter fiber heterogeneity. Magn Reson Med 2002;48:577-82.

Tuch DS, Reese TG, Wiegell MR, Wedeen VJ. Diffusion MRI of complex neural architecture. Neuron 2003;40:885-95.

van den Heuvel M, Mandl R, Luigjes J, Hulshoff Pol H. Microstructural organization of the cingulum tract and the level of default mode functional connectivity. J Neurosci 2008;28:10844-51.

van den Heuvel MP, Mandl RC, Kahn RS, Hulshoff Pol HE. Functionally linked restingstate networks reflect the underlying structural connectivity architecture of the human brain. Hum Brain Mapp 2009;30(10):3127-41.

Van Dijk KR, Hedden T, Venkataraman A, Evans KC, Lazar SW, Buckner RL. Intrinsic functional connectivity as a tool for human connectomics: theory, properties, and optimization. J Neurophysiol 2010;103(1):297-321, Epub 2009 Nov 4.

Vincent JL, Patel GH, Fox MD, Snyder AZ, Baker JT, Van Essen DC, et al. Intrinsic functional architecture in the anaesthetized monkey brain. Nature 2007;447: 83-6.
Watts D, Strogatz S. Collective dynamics of 'small-world' networks. Nature 1998;393:440-2.

Wedeen V. Diffusion aniostropy and white matter tracts. In: Proc. Second Int. Conf. Funct. Mapp. Human Brain; 1996. p. S146.

Wedeen V, Reese T, Tuch D, Weigel M, Dou J, Weiskoff R, et al. Mapping fiber orientation spectra in cerebral white matter with fourier-transform diffusion MR. In: Proc. Intl. Soc. Mag. Res. Med.; 2000. p. 82.

Wedeen VJ, Hagmann P, Tseng WY, Reese TG, Weisskoff RM. Mapping complex tissue architecture with diffusion spectrum magnetic resonance imaging. Magn Reson Med 2005;54:1377-86.

Wedeen VJ, Wang RP, Schmahmann JD, Benner T, Tseng WY, Dai G, Pandya DN, Hagmann P, D’Arceuil H, de Crespigny AJ. Diffusion spectrum magnetic resonance imaging(DSI) tractography of crossing fibers. Neuroimage 2008;41(4):1267-77, Epub 2008 Apr 8.

Wiegell MR, Larsson HB, Wedeen VJ. Fiber crossing in human brain depicted with diffusion tensor MR imaging. Radiology 2000;217:897-903.

Wiggins GC, Polimeni JR, Potthast A, Schmitt M, Alagappan V, Wald LL. 96-Channel receive-only head coil for 3 Tesla: design optimization and evaluation. Magn Reson Med; 2009. www.neurolex.org.

Xydis V, Astrakas L, Drougia A, Gassias D, Andronikou S, Argyropoulou M. Myelination process in preterm subjects with periventricular leucomalacia assessed by magnetization transfer ratio. Pediatr Radiol 2006;36:934-9.

Zhang D, Snyder AZ, Fox MD, Sansbury MW, Shimony JS, Raichle ME. Intrinsic functional relations between human cerebral cortex and thalamus. J Neurophysiol 2008;100:1740-8. 